\title{
Conocimientos y prácticas sobre hábitos de cuidado bucal: más allá de las técnicas
}

\author{
Understanding and practices surrounding oral care habits: \\ beyond techniques \\ Compreensão e práticas em torno dos hábitos de higiene bucal: \\ além das técnicas
}

\section{Daniela Avendaño Vega ${ }^{1}$ Leidy Johanna Gaviria Cadavid ${ }^{2}$ Paulina Díaz Mosquera ${ }^{3}$}

Recibido: 28 de septiembre del 2017

Aprobado: 22 de febrero del 2018

Publicado: 1 de julio de 2019

Cómo citar este artículo:Avendaño Vega D, Gaviria Cadavid LJ, Díaz Mosquera SP. Conocimientos y prácticas sobre hábitos de cuidado bucal: más allá de las técnicas. Rev Nac Odontol. 2019; 15(29):1-15. doi: https://doi.org/10.16925/2357-4607.2019.02.04

Artículo de investigación. https://doi.org/10.16925/2357-4607.2019.02.04

1 Facultad de Odontología, Universidad de Antioquia, Medellín, Antioquia, Colombia ORCID: https://orcid.org/0000-0002-7528-0858

2 Facultad de Odontología, Universidad de Antioquia, Medellín, Antioquia, Colombia ORCID: https://orcid.org/0000-0002-1050-5075

3 Facultad de Odontología, Universidad de Antioquia, Medellín, Antioquia, Colombia ORCID: https://orcid.org/0000-0002-8672-9307

Silvia Paulina Díaz Mosquera

Correo electrónico: diazpaulina1@gmail.com 


\title{
Resumen
}

Introducción: el cuidado de la salud bucal de niños y niñas en la primera infancia está mediado por los conocimientos y prácticas cotidianas de los adultos responsables. Analizar los conocimientos y prácticas de los adultos responsables del cuidado bucal de niños y niñas entre 3 y 5 años vinculados a la Fundación Las Golondrinas, Medellín, Colombia

Materiales y métodos: estudio de caso cualitativo. Se realizaron 27 entrevistas a adultos significativos del cuidado bucal en el entorno de niños y niñas vinculados a la institución.

Resultados y conclusiones: los adultos relatan su posibilidad de acceso a información de diversas fuentes que junto con su motivación y experiencias les ha permitido la construcción de conocimientos y adopción de prácticas para el cuidado bucal en las que encuentran retos y posibilidades para las acciones de cuidado. Como educadores para la salud, es necesario trascender el accionar disciplinar para contribuir a la construcción de procesos protectores en los que se integren los elementos y técnicas de higiene a prácticas contextuadas que aporten a diferentes aspectos de una crianza que cuide de los niños y las niñas en diferentes dimensiones de su ser.

Palabras clave: cuidado del niño, salud bucal, hábitos, conocimientos

\begin{abstract}
Introduction: The oral health care of children in early childhood is mediated by the knowledge and daily practices of responsible adults. This work attempts to analyze the knowledge and practices of adults responsible for oral care of children between 3 and 5 years, linked to the Las Golondrinas Foundation, Medellin, Colombia.

Materials y methods: A qualitative case study, 27 interviews were conducted with significant adults related to the oral care of children linked to the institution.

Results and conclusions: The adults reported their ease of access to information from various sources that, together with their motivation and experiences, has allowed them to build knowledge and adopt oral care practices in which they find new possibilities for care actions. As health educators, it is necessary to transcend disciplinary actions to contribute to the construction of protective processes, in which hygiene elements and techniques are integrated into contextual practices that contribute to different aspects of parenting, taking care of children in their different states of being.
\end{abstract}

Keywords: child care, oral health, habits, knowledge.

\section{Resumo}

Introdução: 0 cuidado em saúde bucal de crianças na primeira infância é mediado pelo conhecimento e práticas cotidianas de adultos responsáveis. Este trabalho procura analisar os conhecimentos e práticas de adultos responsáveis pelo cuidado bucal de crianças entre 3 e 5 anos, vinculados à Fundação Las Golondrinas, Medellín, Colômbia.

Materiais e métodos: Em um estudo de caso qualitativo, foram realizadas 27 entrevistas com adultos significativos, relacionados ao cuidado bucal de crianças vinculadas à instituição.

Resultados e conclusões: Os adultos relataram sua facilidade de acesso às informações de várias fontes que, juntamente com suas motivações e experiências, permitiram construir conhecimentos e adotar práticas de cuidado bucal nas quais encontram novas possibilidades de ações de cuidado. Como educadores em saúde, é necessário transcender ações disciplinares para contribuir com a construção de processos de proteção, nos quais elementos e técnicas de higiene são integrados a práticas contextuais que contribuem para diferentes aspectos da parentalidade, cuidando das crianças em seus diferentes estados de ser.

Palavras-chave: puericultura, saúde bucal, hábitos, conhecimento. 


\section{Introducción}

El presente artículo se deriva de una investigación que tuvo como objetivo general analizar los conocimientos y prácticas de los adultos responsables del cuidado bucal de niños y niñas entre 3 y 5 años que asistían en el año 2014 a la Fundación las Golondrinas en su sede Villa Liliam (FLG), Comuna 8 de Medellín (Colombia). Esta institución hace parte de los operadores que implementan el programa municipal Buen Comienzo (BC) que busca la atención integral de niños y niñas durante sus primeros 5 años de vida, priorizando las familias con mayores condiciones de vulnerabilidad (1).

De acuerdo con el Plan Operativo de Atención Integral a la Primera Infancia de la Fundación (2), para el año 2014 asistían al programa 185 niños y niñas de los cuales el 70 \% estaban en edades entre los 3 y los 5 años. El $100 \%$ de la población pertenecía a los estratos socioeconómicos 1 y 2, de ellos el $23 \%$ vivía en condiciones de hacinamiento y el 19 \% de las familias tenían condición de desplazamiento. Se identificó que de los acudientes, entendidos por la institución como "aquel adulto significativo que acompaña los niños y niñas en los procesos pedagógicos del centro infantil" (3), el 68 \% correspondían a mamás, el 10 \% a abuelos, 3 \% padres y el restante a otros acompañantes como hermanos, tíos y vecinos.

La motivación para este trabajo partió de nuestro interés como odontólogas por aportar al cuidado de niños y niñas en la primera infancia, en el marco de un convenio interinstitucional entre la FLG y la Facultad de Odontología de la Universidad de Antioquia, Colombia, en el que se acompaña a los niños y las niñas de la Fundación en acciones preventivas relacionadas con el componente bucal de la salud, teniendo presente que junto con la gestación, el cuidado en sus primeros años de vida es fundamental para sus condiciones de bienestar presente y futuro (4). Así mismo, la realización del estudio respondió a la intención de contribuir a las reflexiones relacionadas con la garantía y protección integral de los derechos de los niños en un acompañamiento que de acuerdo con la ley de infancia y adolescencia (5) implica la corresponsabilidad de la familia, la sociedad y el Estado.

El cuidado delos niñosy las niñas está mediado por los conocimientosy prácticas de los adultos que los acompañan. Es así como Ochoa (6) y otros afirman que la presencia de adultos significativos es fundamental para contribuir a la ampliación de saberes en los niños que se pueden ver reflejados posteriormente en unas prácticas de autocuidado favorables para la salud. En este estudio se incluyen las agentes educativas institucionales y de manera fundamental los adultos del entorno familiar, entendiendo este con Posada, Gómez y Ramírez (7) como contexto de socialización 
primaria que permite fortalecer los aprendizajes de todos sus integrantes y potenciar experiencias de salud con base en lo cotidiano, el sentido común y la cultura en la cual están inmersos.

Cuidar, como expresa Boff (8) representa una actitud de ocupación, preocupación y compromiso afectivo con el otro que adquiere su importancia en el entorno familiar teniendo en cuenta que las prácticas cotidianas generan vínculos afectivos que incluyen una respuesta a las demandas de cuidado, en una responsabilidad ética que según Duch y Mèlich (9), implica el deseo y disposición de acoger y proteger a otro que, como los niños, se están instalando en el mundo.

Es así como varios estudios señalan la importancia de abordar la relación familia-cuidado en la primera infancia, teniendo en cuenta que en sus hallazgos se encuentra una correlación entre las representaciones y hábitos parentales en la construcción de hábitos en los niños $(10,11)$ y entre el nivel educativo de los padres y el estado de salud bucal en la primera infancia (12). Además se plantea la necesidad de vincular a los adultos significativos en los procesos de promoción de la salud bucal (13) y en la construcción de un cuidado de calidad (14).

Cuidar la salud bucal de los niños en el entorno familiar implica entonces aportar a sus condiciones de bienestar cuando se piensa la boca como espacio de diversas significaciones y experiencias de sí mismo (15) y como territorio (16)de expresión del afecto, la comunicación y apoyo a la nutrición. En este cuidado se incluye la construcción de hábitos para prevenir enfermedades bucales que cobran su importancia para la primera infancia con los resultados del IV Estudio Nacional Salud Bucal (17) en el que se identificó un incremento en la experiencia de caries en los niños de 3 y 5 años en un 47,10 y 62,10 \% respectivamente, hecho que llama a mantener una actitud de alerta por parte de los profesionales de la salud, en relación con la generación de conocimiento que permita acompañar a las familias en el cuidado de la salud bucal de los niños en edades tempranas, de acuerdo a su contexto.

\section{Materiales y métodos}

La intención comprensiva de la investigación llevó a realizar un estudio cualitativo de caso de tipo descriptivo en el que a partir de un número limitado de casos seleccionados, en función del propósito de la investigación, se da cuenta de las complejidades presentes (18), para aportar información básica sobre el tema de investigación (19) y presentar la descripción de un fenómeno en su contexto. En este estudio se tomó como caso el cuidado de la salud bucal de los niños y niñas a partir de sus hábitos 
diarios y de los sentidos que los adultos le otorgan a sus acciones en el contexto de ciudad que habitan, en el marco de un espacio común de pertenencia institucional y en este periodo de tiempo. Así se asume el caso como "un sistema delimitado en tiempo y espacio de actores, relaciones e instituciones sociales" (20), que se refiere a la elección de un objeto y sujetos para participar en la investigación.

Según la Resolución 008430 de 1993 del Ministerio de la Salud y Protección Social de Colombia (21), este estudio se considera de riesgo mínimo. La recolección de la información se realizó en el entorno institucional previo consentimiento verbal y escrito tanto de la institución como de los participantes y se tuvieron como criterios éticos fundamentales una actitud de respeto, escucha y reflexión permanente, así como la devolución de los resultados. De igual manera se garantizó el derecho a la intimidad y anonimato de los participantes. Los testimonios que aparecen en los resultados se identifican de acuerdo con tres criterios: la relación que tiene el adulto entrevistado con el niño o la niña, la edad del niño o niña al momento de la entrevista y entre paréntesis la letra $\mathrm{E}$ que corresponde a entrevista y un número que corresponde al número que se asignó a la misma de acuerdo con el momento de su realización. Adicionalmente el estudio fue revisado y aprobado por el comité de ética de la Facultad de Odontología de la Universidad de Antioquia en su acta número 7, concepto 18-2016.

Como estrategias de reconocimiento de los participantes se revisaron documentos institucionales que daban cuenta de su contexto socioeconómico, y para la recolección de información se realizaron 20 entrevistas a adultos responsables en el entorno familiar cuya inclusión en el estudio estuvo mediada por su relación directa con el cuidado de niños o niñas. También fueron entrevistadas cuatro agentes educativas (AE) responsables de dos grupos de niños y niñas entre 3 y 5 años en la institución, un agente educativo odontólogo y dos coordinadoras del programa. Así fue posible reconocer el entorno de los niños y las niñas en lo relacionado con el cuidado de su salud bucal desde las diversas maneras en que lo asumen los adultos que los acompañan.

En consonancia con el propósito del estudio, el análisis de la información tuvo una intención hermenéutica de lo narrado (22) por los participantes en las entrevistas; y por tanto estas se convirtieron en textos a partir de los cuales se buscaron patrones de convergencias y divergencias que permitieron una agregación categorial (23) y la emergencia de estructuras, lógicas y significados (24) relacionados con el objetivo del estudio y que a través de su organización en mapas dieron sentido a los conocimientos, prácticas y sus relaciones en torno al cuidado de la salud bucal de los niños. El análisis se realizó de manera permanente durante la recolección de la información y para ello se utilizó un procesador de texto en el que se elaboraron las matrices de análisis y los mapas de relaciones. 
Los resultados fueron validados por los participantes en la institución a través de un conversatorio y a partir de éste se diseñó y envió un plegable para las familias pertenecientes al programa con los resultados de la investigación y aportes a sus conocimientos y prácticas cotidianas sobre el cuidado de la salud bucal de niños y niñas.

\section{Resultados}

El cuidado bucal en este contexto incluyó, como se mencionó en la metodología, las voces de diferentes adultos que tienen relación con los niños y niñas participantes. Los resultados que se presentan a continuación se centran en las dinámicas del entorno familiar pues en éste se concretan con mayor diversidad las acciones cotidianas que, en interacción con las rutinas institucionales, tienen influencia en las acciones de cuidado bucal de los niños y las niñas.

Los adultos que se encargan de los momentos de higiene en la institución tienen como guía los compromisos establecidos en los lineamientos del programa Buen Comienzo dentro del pilar de ritmos cotidianos a partir de los cuales se establecen rutinas en las que usan diferentes mediadores pedagógicos para las dinámicas de salud bucal. Los conocimientos que declaran las AE en la institución vienen principalmente de su propia formación universitaria, sus experiencias personales y algunas capacitaciones que realiza el AE odontólogo en el marco del convenio con la FLG. Así mismo, viven el momento de higiene diariamente con la valoración que hacen de la creación del hábito, el suministro de los elementos de higiene necesarios y la flexibilidad que implica el trabajo con niños y niñas en relación con la variabilidad en su comportamiento, el tiempo y el número de momentos que es posible dedicar cada día al cuidado bucal. Los niños y las niñas tienen entonces un espacio de motivación permanente en relación con el cuidado bucal en el espacio institucional en que convergen.

A continuación, se presentan los resultados sobre los conocimientos y el ejercicio de las prácticas relacionadas con los hábitos que aportan al cuidado de la salud bucal en las familias entrevistadas.

Los acudientes entrevistados fueron 10 mamás, 7 abuelas, 2 papás y 1 abuelo de los niños y las niñas. En relación con la edad de los niños que tienen a su cargo, el 50 \% tenían 4 años, 25 \% 5 años y el 25 \% restante tenían 3 años al momento de la entrevista. El orden de nacimiento de los niños era primer hijo para doce niños y segundo para ocho. En lo que se refiere a la afiliación al Sistema General de Seguridad Social en Salud, el $76,4 \%$ de estas familias pertenecían al régimen subsidiado y el $17,6 \%$ al contributivo. 


\section{La construcción de conocimientos para el cuidado de la salud bucal en el entorno familiar}

Los conocimientos sobre cuidado de la salud bucal de los acudientes entrevistados en relación con el fortalecimiento de hábitos cotidianos, se originan en la información que han apropiado de diversas fuentes. Una de ellas es la propia experiencia que algunos relacionan con enfermedades bucales en la familia o con la mayor información o acceso a recursos disponibles para cuidar la salud bucal en comparación con su propia niñez, lo cual los motiva a acoger acciones preventivas.

- Lo llevo a odontología para que no le vuelvan a dar esas picas, y no vaya a quedar como el abuelito que no tiene dientes porque a él le toco fue otra época y no había tanta cosa pero hoy que él tiene cepillo y uno trata es muy importante. Mamá niño 5 años (E20).

-Es que eso es como hereditario, a mí no me gusta el cepillo y he sufrido de muela hueca por las caries, entonces obviamente yo estoy encima pa cepillarlo, para que a él no le pase lo mismo. Mamá niño de 3 años (E12).

-Es difícil ahora con tantas cosas que le ofrecen para los niños, con tanto apoyo ¿cómo para dejarle dañar la dentadura a un niño no? Eso era en el tiempo de uno. Abuela niña de 4 años (E14).

La construcción de conocimientos se concreta así mismo en una motivación para proteger los niños y las niñas de la alteración producida por las enfermedades prevalentes en odontología como la caries y la enfermedad de las encías, en relación con las funciones de masticación y estética, en la que se da gran valoración a la sonrisa y a los dientes, entendidos como parte importante de una buena imagen personal. Además, algunos consideran importante fomentar el hábito como apoyo al fortalecimiento de la autonomía en los niños en relación con su propio cuidado.

-Es muy importante desde niño enseñar a los niños a que se cuiden los dientes porque es que realmente la apariencia de uno siempre es la sonrisa, uno sin sonrisa no es nada. Abuelo niño 2 años (E9). 
—Desde pequeño hay que enseñarle todas esas cualidades para que cuando crezca tenga modo de irse solo, pues que ya uno lo suelte, que ya ellos saben que deben cuidarse la dentadura. Abuela niña de 4 años (E14).

- Lo primordial de la persona es la cara y los dientes, ya el resto del cuerpo lo tiene uno tapado [...] porque usted conversa y lo primero que muestra es la dentadura, si la tiene cepillada, si la tiene mala. Abuela niña 4 años (E16).

Así mismo expresan una construcción de sus conocimientos sobre cuidado bucal en la interacción con otras fuentes representadas en otros miembros de la familia, los medios de comunicación, los programas estatales a los que acceden, los profesionales o instituciones de salud a los que se acercan y la FLG.

-Porque a uno los medios de comunicación le enfatizan que la salud empieza por la boca, y está demostrado que sí, cuando uno tiene una buena higiene oral uno se evita enfermedades, se evita muchas cosas. Papá niña 4 años (E2).

— Lo que yo sé, se lo enseñan a uno chiquito en la casa. Cuando necesito información le pregunto a mi mamá o voy a preguntar en el hospital directamente, allá hay carteles en las paredes con información. Mamá niña 4 años (E11).

— [...] aprendemos acá en la institución (FLG), que están muy pendientes que estemos muy al día con las cosas de los niños. Mamá niña 4 años (E18).

\section{Dinámicas actuales de cuidado bucal en casa}

Los acudientes entrevistados consideran importante la higiene bucal diaria y, por tanto, relatan que mantienen de manera permanente elementos de higiene bucal para hacerlo. En relación con los elementos que usan para el cuidado bucal diario de los niños, resaltan el uso de cepillos dentales para niños, poca o ninguna utilización de la seda dental y en relación con la crema dental existen quienes las utilizan fluoradas, en concentraciones 
de adultos y niños, y cremas dentales sin flúor, identificando que para los niños es poca cantidad y declarando tener información sobre la función de cada uno.

—Nos levantamos, nos cepillamos, él como viene para la escuela acá lo cepillan por segunda vez, y en la casa también se cepilla por ahí hasta las 5 o 6 de la tarde, no utilizamos la seda dental, la crema dental es igual para todos, pero el niño se cepilla solo y escupe todo. Mamá niño de 4 años (E4).

-Yo le pongo la crema en el cepillo porque a veces ella la cogía y lo llenaba de punta a punta y yo, no mami, así no, vea es este poquito que es la mitad y ya ella se cepilla. Abuela niña de 5 años (E15).

- La mamá cuando él acaba de comer lo cepilla, entonces le pasa eso por los dientes (la seda), porque a veces el cepillo no saca todo, saca una parte, pero lo demás no lo saca. Abuela niño 4 años (E5).

En relación con las prácticas reconocen el momento de la mañana y noche como su responsabilidad, pero relatan retos para su realización que se expresan en las condiciones de tamaño de la cavidad bucal de los niños, la negación del niño a que lo acompañe un adulto, la falta de motivación del adulto a asumir la negativa del niño y dificultades relacionadas con el tiempo requerido para cada momento de higiene pues tienen varias responsabilidades en sus hogares o por fuera de él.

- Pues la verdad a mi hijo casi nunca le gusta cepillarse, es una pelea diaria, así que la verdad yo no lo cepillo así que dos o tres veces al día [...] es un cepillaito ahí como peliando con él porque no le gusta, y ya de tanto peliar con él hasta la verdad a mí me da como pereza, entonces a él solo se cepilla por la mañana. Mamá niño de 3 años (E12).

- La verdad es que no uso la seda porque me da miedo aporriarla, es que con esa boquita tan chiquita de pronto la aporreo. Mamá niña 4 años (E11).

_Ella a veces da mucha dificultad para cepillarla, le da pereza, a veces se deja, otras veces no, entonces yo le doy el cepillo para que ella misma se cepille. Abuela niña de 4 años (E16). 
En los testimonios se encuentran también algunos adultos y niños que han tratado de instaurar medidas para remediar las dificultades en el momento de higiene, pensando en cumplir el objetivo de hacerla diariamente:

—Él se levanta, lo desayuno para que venga al colegio, lo baño y así se le va bajando el desayuno y ya cuando le voy a cepillar los dientes no le da tan duro porque antes que lo bañaba primero el hacía como si fuera a vomitar. Mamá niño 5 años (E20).

—Cuando la niña ve que no hay seda, entonces ella me dice: - mamita me da un hilito. Y yo le doy el pedacito de hilo, entonces ella empieza como a saber. Abuela niña de 5 años (E15).

-Ellos por ejemplo cuando ven que uno como adulto coge el cepillo entones también quieren y me toca organizarles el cepillo, y pues se les hecha poquito kolino [crema dental] sí, y usted como sabe que son pequeños es malo como darles mucha crema. Abuela niño 5 años (E19).

El cuidado bucal que realizan los adultos significativos en casa incluye la aplicación de conocimientos adquiridos de diversas fuentes y su aplicación a prácticas que las familias recrean de acuerdo con sus necesidades y posibilidades particulares.

\section{Discusión}

Los conocimientos que construyen los adultos significativos de niños y niñas para acompañarlos a formar sus hábitos tienen gran asiento en la información a la que acceden y el significado que se le da a esta información a partir de las experiencias previas, prácticas cotidianas y la construcción de su responsabilidad en el cuidado bucal de los niños y las niñas.

A partir de los resultados de este estudio se hace evidente una amplia posibilidad de los adultos participantes para obtener información de diversas fuentes que esté relacionada con el cuidado de la salud bucal, a partir de la cual concretan acciones cotidianas en las que está presente, de manera transversal, un sentido de cuidar que incluye pero que está más allá de los elementos y las técnicas para la higiene. Así mismo y en coincidencia con lo encontrado por otros estudios $(25,26)$, los adultos expresan una gran motivación para el cuidado del componente bucal de 
la salud, como rechazo a experiencias previas desagradables, el reconocimiento de un mayor acceso a información y su responsabilidad como cuidadores.

La experiencia, motivación e interacción con otros, como fuentes de información, han permitido a los adultos del entorno familiar hacer reflexiones para la acción que expresan en la toma de decisiones, identificación de dificultades y búsqueda de alternativas a los retos que se presentan a partir de los momentos de higiene. Lo anterior se traduce para algunos en una sensación de ambivalencia en la que existe, por una parte, un sentimiento de motivación, posibilidad y solvencia para identificar los elementos y prácticas necesarios para el cuidado bucal de las niñas y los niños; y por otro, los sentimientos de frustración relacionados con las dificultades en su comportamiento en el momento de la higiene. Lo anterior concuerda con lo encontrado por Mofidi, Zeldin y Rozier (27) quienes exponen en los resultados de su investigación que algunos de los adultos entrevistados mostraban actitudes a la vez positivas y negativas hacia el cuidado de la salud bucal en niños pequeños. Aquí el reto tiene que ver con la posibilidad de los adultos para equilibrar estos sentimientos de forma que el resultado sea satisfactorio para el adulto como cuidador y aporte al afianzamiento de la autonomía, la seguridad y la disciplina para el niño, en una construcción del cuidado como relación recíproca en la que se logre una cooperación sintonizada en términos emocionales y comportamentales (28) y que en su condición de cuidado sensible (29) se ajuste a los estados emocionales y momento evolutivo de el niño o la niña para así favorecer ambientes de cuidado amorosos y cálidos.

Entendiendo la crianza con Rengifo (30) como una conversación afectiva entre equivalentes en la que acompañando a niños y niñas en sus aprendizajes, se transforma y aprende el adulto que cría y que "todas las indicaciones sobre el cuidado y la atención del niño o niña forman parte de la crianza" (31), es posible analizar como educadoras para la salud que el acompañamiento en la concepción y concreción de hábitos en diferentes entornos debe promover un diálogo de saberes, emociones y significados, en el que se valide el conocimiento adquirido por las experiencias de vida tejidas con el niño o la niña, reconociendo así también a los adultos que acompañan.

Es una oportunidad para afirmar que el rol de las educadoras incluye pero no se limita a la información pues ésta es cada vez de más fácil acceso para todos y que las prácticas requieren de una motivación al cuidado y la generación de una empatía con los adultos que están a cargo de los niños y las niñas que parta del reconocimiento de los retos que les implican los momentos de higiene en edades tempranas y que así puedan acercarse a hacerlos con sentido de proceso, con mayor sensación de logro, con posibilidad de reflexión, búsqueda de soluciones y como posibilidad para 
promover momentos de interacción amorosa con los niños y las niñas en su proceso de crianza, que redunden en mejores condiciones de su salud bucal.

En este orden de ideas, los resultados de las entrevistas a los adultos entrevistados permiten pensar en la educación para la salud como una posibilidad para fortalecer el apoyo a las familias a partir de la construcción de procesos protectores entendidos con Breilh (32) como fenómenos favorecedores de la vida humana individual y colectiva y como oportunidad de acompañar que va más allá de las técnicas de higiene.

Para continuar avanzando en estudios que se acerquen a la comprensión de las dinámicas relacionadas con los conocimientos y prácticas de los adultos significativos que tienen a cargo el acompañamiento cotidiano de niños y niñas en diferentes aspectos de su salud, es importante ampliar el conocimiento disponible sobre sus criterios de búsqueda de información y lo que las familias perciben y esperan de los profesionales de la salud en relación con el asesoramiento familiar en diferentes contextos.

\section{Conclusiones}

Los participantes de este proceso evidencian la posibilidad de acceso a información y experiencias que les permiten tener claridad sobre su responsabilidad y sobre las acciones que deben tener en el cuidado de la salud bucal de los niños y las niñas. Lo anterior resalta la importancia de concebir los procesos de acompañamiento desde la educación para la salud desde una perspectiva que trascienda lo informacional, técnico y disciplinar para contribuir a la construcción de procesos protectores en los que se integren los momentos cotidianos de higiene a prácticas contextuadas que aporten a diferentes aspectos de una crianza que cuide de los niños y las niñas en diferentes dimensiones de su ser.

\section{Referencias}

1. Alcaldía. Programa Buen Comienzo [Internet]. Medellín [consultado enero de 2018]. Disponible en: https://medellin.edu.co/buen-comienzo/inicio-buen-comienzo

2. Fundación Las Golondrinas. Plan Operativo de Atención Integral a la Primera Infancia. Caracterización Comuna 8. Creciendo Sanos y Felices. Medellín: La Fundación; 2014. 
3. Fundación Las Golondrinas. Plan Operativo de Atención Integral a la Primera Infancia. Caracterización Comuna 8. Creciendo Sanos y Felices. Medellín: La Fundación; 2014.

4. Roales-Nieto J, Moreno-San Pedro E, Vinaccia E, Contreras F, Fernández H, Londoño X, et al. Hábitos básicos de salud y creencias sobre salud y enfermedad en adolescentes de España, Colombia Y México. Rev Lat Am Psicol. 2004; 36(3):483-504. Disponible en: http://www. redalyc.org/pdf/805/80536309.pdf

5. Colombia. Congreso de la República. Ley 1098 de 2006, por la cual se expide el código de la infancia y la adolescencia. Santa Fe de Bogotá: Diario Oficial No. 46.446.

6. Ochoa EM, Roldán Vargas O, Ramírez BS, Franco AM. Significados del autocuidado bucal para los escolares de instituciones educativas oficiales de Medellín. Revista Nacional de Odontología. 2011; 7(13):40-48. doi: http://dx.doi.org/10.16925/issn.1900-3080

7. Posada-Díaz A, Gómez-Ramírez JF, Ramírez-Gómez H. El niño sano. Una visión Integral. 4a ed. Bogotá: Editorial Medica Panamericana; 2016. 564 p.

8. Boff L. El cuidado esencial. Ética de lo humano. Compasión por la tierra. Madrid: Trotta; 2002. $168 \mathrm{p}$.

9. Duch-Alvarez LL, Melich-Sangrá JC. Escenarios de corporeidad: antropología de la vida cotidiana. Barcelona: Trotta; 2005. 391p

10. Ballester R. ¿Atendemos a la salud integral de los hijos? Análisis de hábitos básicos de salud en menores de 9 años. INFAD Revista de Psicología. 2012; 1(2): 253-262.

11. Escobar-Paucar G, Sosa-Palacio C, Sánchez-Mejía Á. Salud bucal: Representaciones sociales en madres gestantes de una población urbana. Medellín, Colombia. Ciênc. Salud Colectiva. 2011; 16(11): 4 533-4 540. doi: 10.1590/S1413-81232011001200026.

12. Gómez-Osorno AM, Bernal-Álvarez T, Posada-López A, Agudelo-Suárez AA. Caries dental, higiene bucal y necesidades de tratamiento en población de 3 a 5 años de una institución educativa de Medellín y sus factores relacionados. Rev Nac Odontol. 2015; 11(21): 23-35. doi: 10.16925/od.v11i21.933

13. Ochoa-Acosta EM, Roldán-Vargas O, Franco-Cortés AM, Ramírez-Puerta BS, Mejía-Pineda OL. El ambiente escolar y la educación para la salud bucal en instituciones educativas oficiales. Rev Nac Odontol. 2014; 10(19): 47-54. doi: http://dx.doi.org/10.16925/od.v10i19.847 
14. Carbonell OA. La sensibilidad del cuidador y su importancia para promover un cuidado de calidad en la primera infancia. Cienc.Psicol. 2013; 7(2): 201-207. Disponible en: http://www. redalyc.org/articulo.oa?id=459545415008

15. Malagon-Oviedo R. La boca como representación. En: Viveros-Vigoya M, Garay-Ariza G, compiladoras. Cuerpo, diferencias y desigualdades. Bogotá: Centro De Estudios Sociales. Facultad de Ciencias Humanas Universidad Nacional de Colombia; 1999.

16. Botazzo C. El nacimiento de la odontología: una arqueología del arte dental. Buenos Aires: Lugar; 2010. 208p.

17. Ministerio de Salud y Protección Social. Cuarto Estudio Nacional de salud Bucal. Bogotá: Centro Nacional de Consultoría; 2014. 197 p.

18. Kazez R. Los estudios de casos y el problema de la selección de la muestra: Aportes del sistema de matrices de datos. Subjetividad y procesos cognitivos [Internet]. 2009 [consultado 8/10/2017]. 71-89 p. Disponible en: http://www.scielo.org.ar/scielo.php?script= sci_arttext\&pid=S1852-73102009000100005\&lng=es\&tlng=es.

19. Galeano-Marín ME. Estrategias de investigación social cualitativa. El giro en la mirada. Medellín: La Carreta; 2004. 239 p.

20. Neiman G, Quaranta G. Los estudios de caso en la investigación sociológica. En Vasilachis de Gialdino I, coordinadora. Estrategias de investigación cualitativa. Barcelona: Gedisa; 2006. $275 \mathrm{p}$.

21. Colombia. Ministerio de Salud y Protección Social. Resolución 08430 de 1993. Por la cual se establecen las normas científicas, técnicas y administrativas para la investigación en salud. Bogotá; 1996.

22. De Souza-Minayo MC. La artesanía de la investigación cualitativa. Buenos Aires: Lugar; 2009. $336 \mathrm{p}$.

23. Creswell J. Qualitative inquiry and research design. Choosing among five approaches. California: SAGE; 2012. 448 p.

24. Galeano-Marín ME. Diseño de proyectos en la investigación cualitativa. Medellín: Fondo Editorial Universidad EAFIT; 2004. 82 p. 
25. González-Martínez F, Hernández-Saravia L, Correa-Mulett K. Representaciones sociales sobre higiene bucal en madres y cuidadores de hogares infantiles. Rev Cubana Salud Pública. 2013; 39(1):59-68. Disponible en: http://scielo.sld.cu/scielo.php?script=sci_arttext \&pid=S0864-34662013000100006\&lng=es.

26. Gómez-Arias MY, Franco-Londoño LY, Gaviria-Torres JM, Baena-Muñoz LM, Ortiz Restrepo JF, Zapata-Bedoya JP, Bernal-Álvarez T, Agudelo-Suarez AA. Evaluación del componente bucal. Proyecto "Escuelas Saludables" de la Fundación las Golondrinas, Medellín, 2006-2011. Rev Nac Odontol. 2013; 9(16): 15-2. doi: 10.16925/od.v9i16.6

27. Mofidi M, Zeldin L, Rozier G. Oral Health of Early Head Start Children: A Qualitative Study of Staff, Parents, and Pregnant Women. American Journal of Public Health. 2009; 99(2):245-251. doi: https://dx.doi.org/10.2105\%2FAJPH.2008.133827

28. Carbonell OA. La sensibilidad del cuidador y su importancia para promover un cuidado de calidad en la primera infancia. Cienc Psicol. 2013; 7(2): 201-207. Disponible en: http://www. redalyc.org/articulo.oa?id=459545415008

29. Carbonell OA, Posada G, Plata SJ, Mendez S. Las relaciones vinculares: Un contexto para potenciar el derecho al bienestar de niños y niñas. Cuadernos de Psicologia. 2005 (1): 31-38.

30. Rengifo, G. La enseñanza de estar contento. Educación y afirmación cultural andina. Lima: PRATEC; 2003. 198 p.

31. PeñarandaF.Lacrianzacomocomplejo histórico, socioculturalyontológico:unaaproximación sobre educación en salud. Rev Latinoam Cienc Soc Niñez Juv. 2003; (9):945-956. Disponible en: http://www.scielo.org.co/scielo.php?script=sci_arttext\&pid=\$1692-715X2003000100008

32. Breilh J. Epidemiología Crítica, Ciencia emancipadora e interculturalidad, Buenos Aires: Lugar; 2003. 320 p. 